\title{
Acoustofluidic control of bubble size in flow-focusing configuration ${ }^{\dagger}$
}

\author{
Zhuang Zhi Chong, ${ }^{a}$ Shu Beng Tor, ${ }^{a}$ Ngiap Hiang Loh, ${ }^{a}$ Teck Neng Wong, ${ }^{* a}$ Alfonso M Gañán- \\ Calvo, ${ }^{b}$ Say Hwa Tan, ${ }^{a}$ Nam-Trung Nguyen, ${ }^{* c}$
}

\author{
Received Xth $X X X X X X X X X X 20 X X$, Accepted Xth $X X X X X X X X X 20 X X$ \\ First published on the web Xth $X X X X X X X X X X 20 X X$ DOI: 10.1039/b000000x
}

In this paper, we demonstrate a method to control the generated bubble size in flow-focusing configuration using an ultrasonic transducer. We induce acoustic streaming using a forward moving, oscillating gas-liquid interface at vibration mode of the transducer system. The induced streaming has substantial effect to the process of bubble formation, which result in significant increment in gas flow rate and generated bubble size. This method is applicable to variable flow conditions: gas pressure from 30 to $90 \mathrm{kPa}$, flow rate from 380 to $2700 \mu \mathrm{L} / \mathrm{h}$. The increment in the generated bubble size is repeatable in seconds. We believe this method will enhance the capability of microfludic bubble generator to produce wide range of bubble size.

\section{Introduction}

Micron size bubbles are used in numerous applications such as ultrasonic scanning, ${ }^{1}$ target drug delivery, ${ }^{2}$ bubble-triggered drop formation, ${ }^{3}$ liquid slug separation, ${ }^{4}$ fluorination ${ }^{5}$ and hydrogenation. ${ }^{6}$ We can use microfluidic devices of different designs (flow focusing, ${ }^{7}$ T-junction, ${ }^{8}$ and co-flowing ${ }^{9}$ ) to generate monodisperse bubbles. Generally, the control of the bubble size using these devices is limited, as it is constrained by the fluid flow rate, fluid pressure and the geometry of the channel. Unlike bubble generation, droplet generation has many methods developed to achieve better size control. The methods could be acoustic ${ }^{10}$, electric, ${ }^{11}$ thermal ${ }^{12}$, magnetic ${ }^{13}$ and mechanical. ${ }^{14}$ There is a need to address this capability gap between bubble generation and droplet generation.

Bubbles expand and contract with the acoustic pressure rarefaction and compression. ${ }^{15}$ When driven by periodic acoustic pressure change from ultrasonic transducer, bubbles rectify rapid oscillation motion of the gas-liquid interface into steady streaming flow in the liquid around the bubbles. ${ }^{16}$ This acoustic streaming is of Rayleigh type, which is different from quartz wind streaming, where the later operates in the $\mathrm{MHz}$ range. ${ }^{17,18}$ The streaming flow has been used in microfluidic devices for micromixing, ${ }^{19,20}$ micropumping, ${ }^{21}$ microparticle sorting ${ }^{22}$ and sonoporation of suspended cells. ${ }^{23}$

The bubbles that induces Rayleigh streaming in microflu-

${ }^{a}$ School of Mechanical and Aerospace Engineering, Nanyang Technological University, 50 Nanyang Avenue, Singapore 639798

${ }^{b}$ de Fluidos e Ingeniería Aeroespacial, Universidad de Sevilla, E-41092 Sevilla, Spain.Email: amgc@us.es

c QLD Micro- and Nanotechnology Centre, Nathan campus, Griffith University, 170 Kessels Road QLD 4111, Australia. Email: namtrung.nguyen@griffith.edu.au

$\dagger$ Electronic Supplementary Information (ESI) available: [01.mp4: Vibrating gas-liquid interface captured at 192440 FPS; 02.avi: Turning on and off in 2 second captured at low shutter speed] idic devices are typically formed by passing liquids along blind side channels. ${ }^{19}$ These semicylindrical air bubbles are difficult to form with desired size as suitable surface properties, blind side channels geometry and initial flow condition are required. Furthermore, bubble size will change after a period of continuous oscillation. Once the device has failed to create or maintain a bubble with desired size, we will need to replace or redesign it.

Instead of forming and trapping bubbles in blind side channels with challenging repeatability, our setup induces streaming using a forward moving gas-liquid interface. The interface moves forward as a bubble is being created. The induced streaming from a forward moving and oscillating gas-liquid interface alters the behavior of bubble formation and thus its generated bubble size. This offers a new and novel way to control the generated bubble size while maintaining the fluid flow rate or pressure.

Our acoustofluidic control of bubble size is easy to implement on microfluidic bubble generator. The ultrasonic transducer used in the setup is available on the market for liquid level measurement, object detection and remote control applications. This setup is efficient too, as the electric energy input to the transducer is lesser than $1 \mathrm{~W}$.

\section{Materials and methods}

We fabricate a polydimethylsiloxane (PDMS) device with a cross-junction channel (width $\mathrm{w}=43 \mu \mathrm{m}$, height $\mathrm{h}=100 \mu \mathrm{m}$ ) using standard soft lithography techniques. ${ }^{24}$. We apply the bottom part of the device with a thin layer of ultrasound gel (ZG-F, General Electric) before attaching it with an ultrasonic transducer (328ET250, Prowave) as shown in Fig. 1. We align the transducer to be concentric to center of the cross-junction channel. 


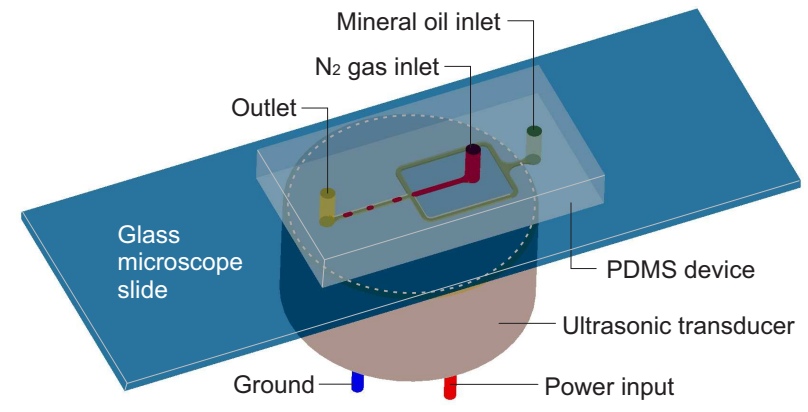

Fig. 1 Bubble generation device with ultrasonic transducer. The schematic is not drawn to the scale.

We form bubbles in oil by flowing nitrogen gas to the central channel of the cross junction and mineral oil (M5904, Sigma Aldrich; viscosity $\eta=30 \mathrm{mPa} \mathrm{s}$; surface tension $\sigma$ $=33 \mathrm{mN} / \mathrm{m}$ ) to the two side channels. We control the volumetric flow rate of the oil using a syringe pump (neMESYS Low Pressure, Cetoni) and maintain the pressure of the gas using a pressure controller (PPC4, Fluke). We observe and capture the bubble generation using a microscope (BX51, Olympus) fitted with a high-speed camera (Miro M310, Phantom). We measure the bubble dimensions and generation frequency from recorded videos using a self-written OpenCV based image processing software.

We use an alternating current (AC) source to power the transducer. The source is produced by amplifying a sinusoidal voltage (33520A, Agilent) using a circuit board with an operational amplifier chip (OPA552, Texas Instruments). We use $\mathrm{AC}$ frequency ranges from 1 to $400 \mathrm{kHz}$ and amplitude up to $40 \mathrm{~V}$ (peak-to-peak voltage) in our experiment. As the transducer heats up during the operation, we use a fan (MCFJ01BM05-9, Toshiba) to prevent the temperature at the junction from increasing more than $2^{\circ} \mathrm{C}$.

\section{Results and discussion}

We generate monodisperse bubbles under gas pressure of $45 \mathrm{kPa}$ and oil flow rate of $960 \mu \mathrm{L} / \mathrm{h}$. After which, we monitor the generated bubble size when the transducer is powered by $\mathrm{AC}$ at variable of frequencies. We observe a significant increase in generated bubble size when the frequency of the $\mathrm{AC}$ is tuned to $128 \mathrm{kHz}$ and $290 \mathrm{kHz}$. Fig. 2(a) shows the frequency response of the generated bubble size from 120 to $136 \mathrm{kHz}$, which has a sharp peak at $128 \mathrm{kHz}$.

We then video capture the process of the bubble generation in 192,440 frames per second when the transducer is powered by $\mathrm{AC}$ of $40 \mathrm{~V}$ at $128 \mathrm{kHz}$. From the video $\left(01 . \mathrm{mp} 4^{\dagger}\right)$, we notice an oscillatory motion of gas-liquid interface. The speed of the motion is much faster than the forward movement of the
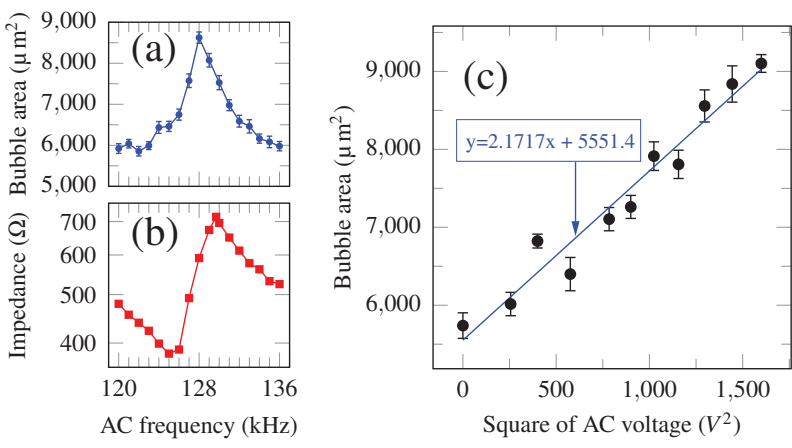

Fig. 2 (a) Bubble size response and (b) electrical impedance of transducer when applied with AC frequency from 120 to $136 \mathrm{kHz}$ (c) Bubble size response when applied with AC of variable voltages at $128 \mathrm{kHz}$. The blue straight line is a linear fitting to the scattering points. The flow condition of bubble generation in (a-c) is $P=45 \mathrm{kPa}, Q_{l}=960 \mu \mathrm{L} / \mathrm{h}$.

gas-liquid interface. The interface oscillates according to the first mode as no nodes is observed in the oscillating interface.

We believe $128 \mathrm{kHz}$ is not the resonant frequency of the gas-liquid interface. Firstly, resonant frequency of the gasliquid interface depends on its radius of curvature and the fluidic pressure, as proven in the solution from Rayleigh-Plesset equation. ${ }^{25}$ If the interface is oscillating at resonant frequency, we should see vigorous oscillation only at a certain period of time. This is because the resonant frequency changes from time to time as the radius of the gas-liquid interface curvature changes throughout the bubble generation process. However, from the video, the interface oscillates with similar amplitude throughout the whole process. Next, the frequency should increase when the oil flow rate and gas pressure is increased if it is to oscillate at resonant frequency. Yet, from the experiment, $128 \mathrm{kHz}$ is still the frequency that we observe largest increment in generated droplet size when we increase the oil flow rate up to $2700 \mu \mathrm{L} / \mathrm{h}$ and the gas pressure up to $90 \mathrm{kPa}$.

We think the frequency is the mechanical vibration mode of the transducer system. We verify this by measuring the electrical impedance of the transducer system, as a pair of electrical resonance and antiresonance (local minimum and maximum impedance magnitude) is usually found around a mechanical vibration mode of piezoelectric actuators. ${ }^{26}$ From the measurement on the attached transducer, the pair do exist around $128 \mathrm{kHz}$, as shown in Fig. 2(b). We also find another pair around $290 \mathrm{kHz}$. Therefore, it is deduced that the increment of bubble size occurs at the vibration modes of the transducer system.

In another test, we monitor the size of the bubble generated under the same flowing condition with AC power of incrementing voltage at $128 \mathrm{kHz}$. As shown in Fig. 2(c), bubble area increases linearly with the square of the applied AC volt- 

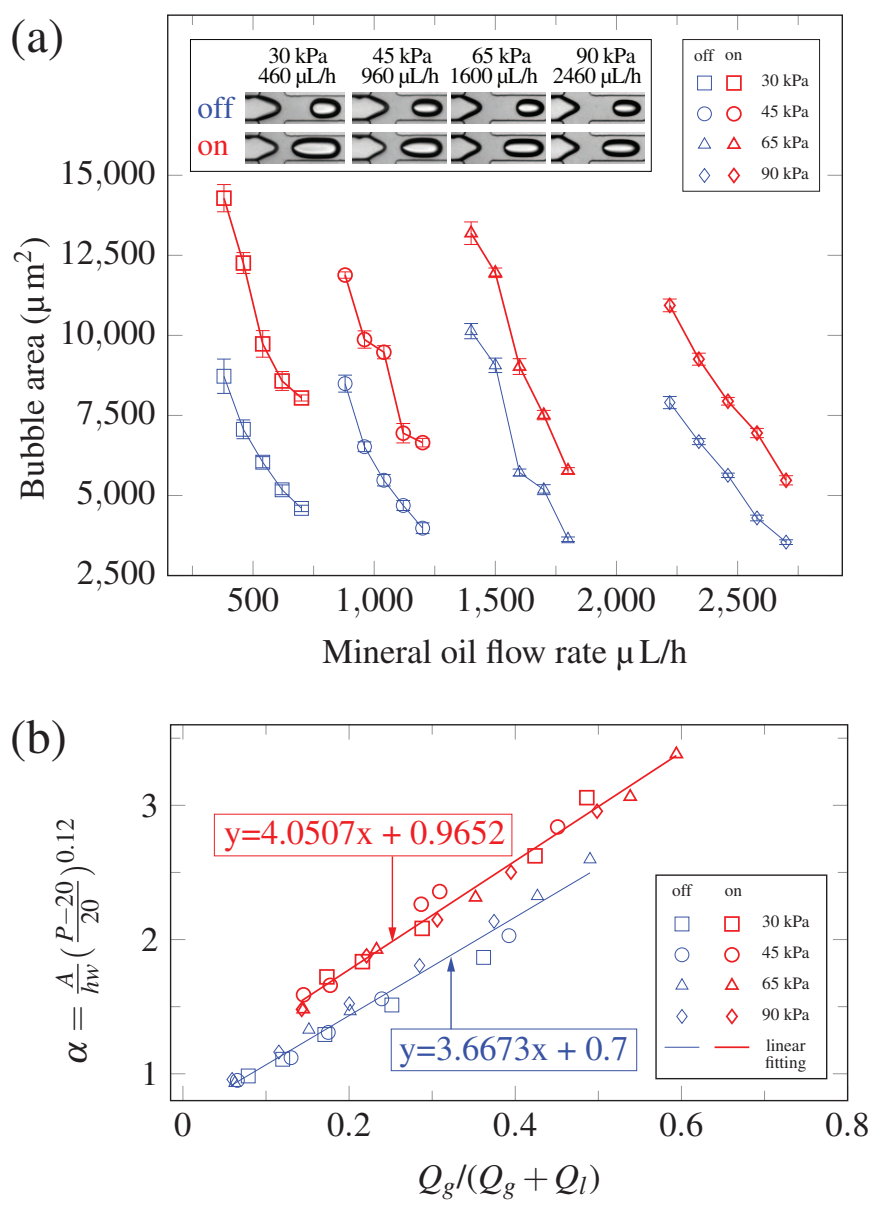

Fig. 3 (a) Generated bubble size under different flowing conditions and transducer states. The insets show the freshly generated bubbles under the selected flowing condition. (b) Results plotted using dimensionless number

age. The increment of the bubble area is directly proportional to the electric energy input to the transducer.

We then evaluate the effect of transducer powered by AC of $40 \mathrm{~V}, 128 \mathrm{kHz}$ on bubble generations under variable of pressures and flow rates. As shown in Fig. 3(a), the area of the generated bubble increases significantly after turning on the transducer for all the tested flowing conditions. The insets in Fig. 3(a) show the freshly generated bubbles under selected flowing conditions for each transducer state.

The result is also plotted in Fig. 3(b) using dimensionless numbers ${ }^{27}$ by using the following relationship,

$$
\alpha \propto \frac{Q_{g}}{Q_{g}+Q_{l}}, \alpha=\frac{A}{h w}\left(\frac{P-P_{\min }}{P_{\min }}\right)^{0.12},
$$

where symbols $\alpha, Q_{g}$, and $Q_{l}$ are dimensionless bubble area, gas volumetric flow rate and liquid volumetric flow rate respectively. Symbols that are used to derive $\alpha$ include 2 vari-

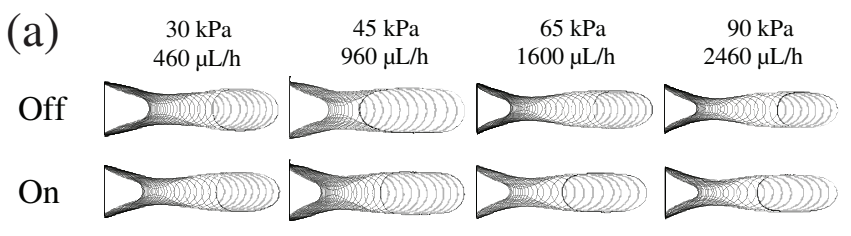

(b)

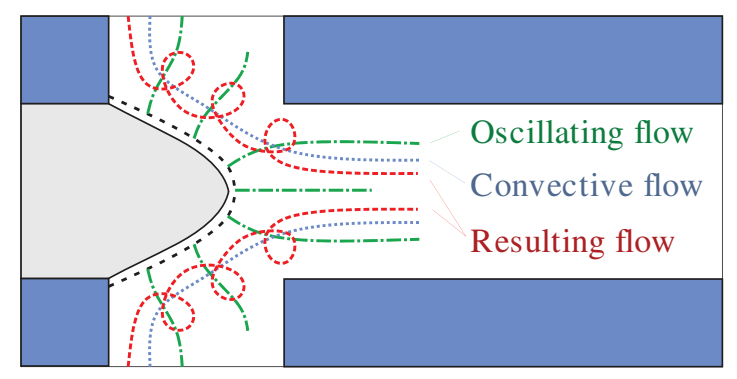

Fig. 4 (a) The time sequence of the expansion of gas-liquid interface during bubble formation (b) Suggested streaming path resulting from the combination of convective flow and oscillating flow

ables (A for bubble area, $\mathrm{P}$ for gas pressure in $\mathrm{kPa}$ ) and 2 constants ( $h$ and $\mathrm{w}$ for channel height and width respectively). The second term in the derivation of $\alpha$ is a small correction factor for pressure, where $P_{\min }$ is the minimum pressure (20 $\mathrm{kPa})$ to achieve stable bubble formation and 0.12 is an arbitrary correction number. Gas flow rate, $Q_{g}$ is calculated from frequency of bubble generation and the average volume of bubble. The volume of a bubble is estimated using the equation mentioned by van Steijn et al. ${ }^{28}$ From Fig. 3(b), gas flow rate increases when transducer is turned on. The generated bubble area converges to a line above the turned off stage line with a similar gradient.

The increment in the generated bubble size upon the activation of the transducer can be related to the Rayleigh streaming formed around the gas-liquid interface. This streaming is rectified from rapid oscillation of the interface driven by the utlrasonic transducer, ${ }^{16}$ with $u_{s} \sim 2 \pi f \varepsilon^{2} a$ as the theoretical streaming velocity. ${ }^{22}$ At $Q_{l}=960 \mu L / h$ and $P=45 \mathrm{kPa}$, the theoretical streaming velocity is $\sim 26 \mathrm{~mm} / \mathrm{s}(\varepsilon=0.04$, $a=20 \mu \mathrm{m}, f=128 \mathrm{kHz}$ ). This is comparable to the average oil flow velocity of $62 \mathrm{~mm} / \mathrm{s}$. The streaming is in RayleighNyborg-Westervelt regime, ${ }^{29}$ as the streaming Reynolds number $\left(R e_{s} \equiv 2 \pi f \varepsilon^{2} a^{2} / v\right)$ is $\sim 0.014$, which is much lesser than 1 in oil medium.

However, the streaming formed around the forward moving, oscillating gas-liquid interface in fast flowing medium can be very complex. Unless detailed experiment is done, we do not have sufficient knowledge to predict and model the streaming accurately. In this paper we could only postulate the existence of induced streaming around the oscillating interface, with its velocity is in the order comparable to the flow velocity.

As shown in the contours tracing the expansion of gas- 


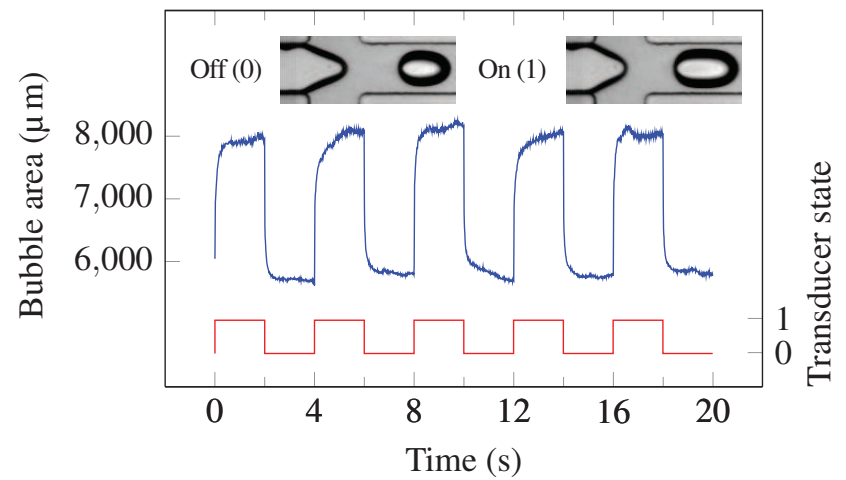

Fig. 5 Generated bubble size response to the transducer state (1 for on, 0 for off). Insets shows the images from capturing at off ( 0 ) state and on (1) state.

liquid interface under variety of situations (Fig. 4(a)), the contours around the interface appears thicker when the transducer is turned on. This shows the streaming effect have caused the interface to expand more. We think the streaming could be a resulting flow combining convective and oscillating flow, as illustrated in Fig. 4(b). This induced streaming is significant enough to influence the process of bubble formation, which increases both the flow rate of the gas and the size of bubble.

Next, we carry out a test to find out the repeatability of the generated bubble size to the transducer state under a constant flowing condition ( $\left.\mathrm{P}=45 \mathrm{kPa}, Q_{l}=960 \mu \mathrm{L} / \mathrm{h}\right)$. In this test, the transducer is switched on and off every 2 seconds. The test result is shown in Fig. 5, where 1 and 0 is the transducer on and off state respectively. The bubble size response to the transducer state is repeatable. The graph shows a sharp rise or fall in the bubble area around switching point of time. We also observe a fast changing of the gas-liquid interface upon the switching of the transducer state. This change is especially obvious when the test is recorded using a lower shuttle speed video $\left(02 . \mathrm{mp} 4^{\dagger}\right)$. This shows the induced streaming is formed very quickly once the transducer is turned on.

\section{Conclusions}

We have demonstrated a method to control the generated bubble size in flow-focusing configuration using an ultrasonic transducer. We induced acoustic streaming using a forward moving, oscillating gas-liquid interface at vibration mode of the transducer system. The induced streaming affected to the process of bubble formation. The gas flow rate and generated bubble size increased significantly when the transducer is turned on. This method was applied and had same effect to variable flow conditions: gas pressure from 30 to $90 \mathrm{kPa}$, flow rate from 380 to $2700 \mu \mathrm{L} / \mathrm{h}$. The increment in the generated bubble size was repeatable in seconds. We believe this method will enhance the capability of microfludic bubble generator to produce wide range of bubble size.

\section{Acknowledgments}

S.H. Tan \& T.N. Wong gratefully acknowledge the research support from the Singapore Ministry of Education (MOE) Tier 2 Grant (No. 2011-T2-1-0-36). We also gratefully acknowledge insightful discussion with Dr. Claus-Dieter Ohl at Nanyang Technological University, Singapore.

\section{References}

1 Enhancing the Role of Ultrasound with Contrast Agents, ed. R. Lencioni, Springer Milan, Milano, 2006.

2 J. M. Tsutsui, F. Xie and R. T. Porter, Cardiovascular ultrasound, 2004, $2,23$.

3 A. R. Abate and D. a. Weitz, Lab on a chip, 2011, 11, 1713-6.

4 V. Linder, S. K. Sia and G. M. Whitesides, Analytical chemistry, 2005, 77, 64-71.

5 R. D. Chambers, D. Holling, R. C. Spink and G. Sandford, Lab on a chip, 2001, 1, 132-7.

6 Science (New York, N.Y.), 2004, 304, 1305-8.

7 A. M. Gañán Calvo and J. M. Gordillo, Physical Review Letters, 2001, 87, 274501.

8 P. Garstecki, M. J. Fuerstman, H. a. Stone and G. M. Whitesides, Lab on a chip, 2006, 6, 437-46.

9 R. Xiong, M. Bai and J. N. Chung, Journal of Micromechanics and Microengineering, 2007, 17, 1002-1011.

10 L. Schmid and T. Franke, Lab on a chip, 2013, 13, 1691-4.

11 H. Kim, D. Luo, D. Link, D. a. Weitz, M. Marquez and Z. Cheng, Applied Physics Letters, 2007, 91, 133106.

12 N.-T. Nguyen, T.-H. Ting, Y.-F. Yap, T.-N. Wong, J. C.-K. Chai, W.-L. Ong, J. Zhou, S.-H. Tan and L. Yobas, Applied Physics Letters, 2007, 91 , 084102.

13 S.-H. Tan, N.-T. Nguyen, L. Yobas and T. G. Kang, Journal of Micromechanics and Microengineering, 2010, 20, 045004.

14 S.-K. Hsiung, C.-T. Chen and G.-B. Lee, Journal of Micromechanics and Microengineering, 2403-2410.

15 K. Ferrara, R. Pollard and M. Borden, Annual review of biomedical engineering, 2007, 9, 415-47.

16 C. Wang, S. V. Jalikop and S. Hilgenfeldt, Biomicrofluidics, 2012, 6, 12801-1280111.

17 N. Riley, Theoretical and Computational Fluid Dynamics, 1998, 10, 349 356.

18 N. T. Nguyen and R. M. White, IEEE transactions on ultrasonics, ferroelectrics, and frequency control, 2000, 47, 1463-71.

19 D. Ahmed, X. Mao, B. K. Juluri and T. J. Huang, Microfluidics and Nanofluidics, 2009, 7, 727-731.

20 Biosensors \& bioelectronics, 2012, 35, 37-43.

21 A. R. Tovar and A. P. Lee, Lab on a chip, 2009, 9, 41-3.

22 C. Wang, S. V. Jalikop and S. Hilgenfeldt, Applied Physics Letters, 2011, 99, 034101.

23 S. L. Gac, E. Zwaan, A. van den Berg and C.-D. Ohl, Lab on a chip, 2007, 7, 1666-72.

24 D. C. Duffy, J. C. McDonald, O. J. Schueller and G. M. Whitesides, Analytical chemistry, 1998, 70, 4974-84.

25 T. Leighton, The acoustic bubble, Academic Press, San Diego London, 1994. 
26 J. Pons, Emerging Actuator Technologies, John Wiley \& Sons, Ltd, Chichester, UK, 2005.

27 T. Cubaud, M. Tatineni, X. Zhong and C.-M. Ho, Physical Review E, 2005, 72, 037302.

28 V. van Steijn, C. R. Kleijn and M. T. Kreutzer, Lab on a chip, 2010, 10, 2513-8.

29 S. J. Lighthill, Journal of Sound and Vibration, 1978, 61, 391-418. 